\title{
Comment parler de l'ETA ? Lecture des ouvrages de Jean Chalvidant, Antonio Elorza et Fernando Reinares
}

Jean-Marie Izquierdo

\section{(2) OpenEdition Journals}

Édition électronique

URL : http://journals.openedition.org/conflits/2045

DOI : $10.4000 /$ conflits.2045

ISSN : 1777-5345

Éditeur :

CCLS - Centre d'études sur les conflits lilberté et sécurité, L'Harmattan

Édition imprimée

Date de publication : 1 mars 2006

Pagination : 173-177

ISBN : 2-296-00622-1

ISSN : 1157-996X

Référence électronique

Jean-Marie Izquierdo, «Comment parler de l'ETA ? Lecture des ouvrages de Jean Chalvidant, Antonio Elorza et Fernando Reinares », Cultures \& Conflits [En ligne], 61 | printemps 2006, mis en ligne le 17 mai 2006, consulté le 30 mars 2021. URL : http://journals.openedition.org/conflits/2045 ; DOI : https:// doi.org/10.4000/conflits.2045

Ce document a été généré automatiquement le 30 mars 2021

Creative Commons License 


\title{
Comment parler de l'ETA ? Lecture des ouvrages de Jean Chalvidant, Antonio Elorza et Fernando Reinares
}

\author{
Jean-Marie Izquierdo
}

1 CHALVIDANT Jean, ETA, l'enquête, Le Coudray-Macouard, Editions Cheminements, 2003, 426 pages.

2 ELORZA Antonio (dir.), ETA, une histoire (Titre original: La Historia de ETA), Paris, Editions Denoël, 2002, 477 pages.

3 REINARES Fernando, Patriotas de la muerte. Quiénes han militado en ETA y por qué, Madrid, Taurus Ediciones, 2001, 207 pages.

4 ETA, l'enquête. Le titre semble sans appel. Voilà qui résonne comme un bilan qui se veut complet et définitif. Voilà qui appellerait à une fin annoncée d'un mouvement terroriste que l'on sait chaque jour plus exsangue, épuisé et isolé. Son titre fait curieusement écho, par ce désir d'exhaustivité, à celui dirigé par Antonio Elorza, ETA, une histoire. L'auteur de cet ouvrage, Jean Chalvidant, ancien reporter pour RMC, est aujourd'hui collaborateur à l'Institut de criminologie de l'Université Paris II-PanthéonAssas. La quatrième de couverture, elle aussi, donne le ton : "Pour la première fois sont révélés dans ce livre de référence cinquante ans de la vie secrète du mouvement séparatiste basque, grâce à un luxe de faits, de précisions, de noms, d'éclaircissements sur les événements qui ensanglantent l'Espagne depuis l'automne du franquisme». Or, d'entrée, le lecteur est plongé dans un opuscule-type des années 1970, dans ces livres traitant de sujets sensibles mêlant sympathie et critique.

5 Ainsi, dès les premières pages, l'ouvrage rappelle un travail de journaliste dont la liberté de ton laisse dubitatif quant à la qualité de l'analyse. Pourtant, l'auteur connaît, sans aucun doute, son sujet. Il resitue l'ETA dans les événements de l'actualité. Il la compare avec celle des premières années, 
retraçant succinctement son histoire, et jouant sur la corde sensible, encombrant le texte de nombreuses anecdotes au style trop facile. Dans un deuxième chapitre, il cherche à situer le conflit géographiquement, ainsi que dans sa trajectoire historique. Que représente l'ETA pour les citoyens basques? Euskadi, réel ou virtuel ? ETA est-il profitable au Pays basque? Le texte se perd en conjectures, surtout, il ne donne pas au lecteur les éléments réellement nécessaires pour situer les faits dans l'histoire de l'Espagne et du Pays basque (précisons que l'absence de table des matières nuit à la compréhension de l'ouvrage). S'arrêtant plutôt sur les excès verbaux des uns et des autres, sans véritablement expliquer qui y recourt et quel est l'effet recherché par l'utilisation d'un tel champ lexical, le discours devient difficile. Les enjeux personnels et partisans ne sont pas réellement présentés.

Dans le troisième chapitre, J. Chalvidant évoque l'ETA depuis l'intérieur. Cette partie, nourrie de multiples exemples, montre bien qui postule à intégrer l'ETA et comment les personnes en question y parviennent. Les logiques ne sont pas véritablement formulées bien que certains titres soient explicites (" de la Kale borroka à la vie clandestine »), la priorité est donnée à l'exemple... D'autres, dont Fernando Reinares, avaient déjà entrepris d'en expliquer les motivations de manière moins dilettante... J. Chalvidant aborde ensuite la situation actuelle de l'ETA. Il décrit les soutiens sociaux et politiques, les relais politiques de l'idéologie de l'ETA, c'est-à-dire le Mouvement de libération national basque (MLNV), mais cela est fait de manière très succincte, restreinte à l'actualité la plus récente. La dernière partie, qui représente près de la moitié de l'ouvrage (225 pages), offre une imposante - et plutôt rare - base de données: les commandos sont répertoriés, les biographies et surtout les interpellations d'un grand nombre de membres de l'ETA (150) sont mises à notre disposition... Voilà peut-être l'origine réelle du titre, ETA, l'enquête, car l'intérêt de ce livre se trouve presque exclusivement dans ce travail de compilation des hommes de l'ETA. Or, ceci ne peut s'adresser qu'à un public averti ou féru de mouvements terroristes. La fin de l'ouvrage donne l'impression d'un road book illustrant un travail certain mené durant de nombreuses années par un auteur passionné par la question. Riche en anecdotes, fourni en données factuelles, cet ouvrage n'en reste pas moins incomplet. Il lui manque trop d'explications resituant les événements dans leur contexte pour faire véritablement sens.

Dans l'ouvrage qu'il coordonne, Antonio Elorza cherche, quant à lui, à donner les pistes explicatives qui manquent au livre de J. Chalvidant. Pourtant, dès l'introduction d'ETA, une histoire, il adopte un parti pris, pas forcément contre la violence de l'ETA mais surtout contre le nationalisme basque en général. En effet, pour expliquer le conflit basque, A. Elorza, qui enseigne les sciences politiques à l'Université Complutense de Madrid, préfère parler d'une forme d'atavisme de la violence propre aux Basques. Il préfère suggérer une incompatibilité viscérale à tolérer l'Espagnol... Ainsi les nationalistes basques sont-ils décrits comme cyniques et réfléchis et conduiraient, main dans la main, la vie politique basque-espagnole depuis des décennies... Parce que ses analyses souffrent d'un style et de propos pamphlétaires et acrimonieux, elles en perdent leur vertu explicative. Les autres contributeurs pondèrent 
heureusement ces dérives anachroniques. Gurutz Jáuregui, juriste et ancien consultant de l'ONU, nous offre une lecture historique plus pertinente. Il s'applique à retracer l'évolution idéologique de l'ETA dans ses contextures historiques. Il revient sur l'importance de la construction nationale espagnole dans l'expression d'une idéologie nationaliste basque. G. Jáuregui défend surtout une tautologie essentielle pour comprendre la naissance puis le développement de l'ETA : c'est la violence du régime franquiste qui permet son éclosion. Ensuite, contre toute attente de la part de l'ETA, la tribune nationale et internationale qu'offre le procès de Burgos l'institutionnalise, sans qu'elle puisse répondre pour autant à son avenir politique en dehors de l'exercice de la violence. José María Garmendia, historien et ancien militant de l'ETA, vient compléter l'analyse. Il offre une vision de "l'intérieur», empreinte d'expériences, démontrant la fragilité de l'organisation à ses débuts. Il précise ainsi les motivations contingentes, dues au contexte répressif et idéologique des années 1960 et 1970. Le développement de l'ETA ne peut définitivement pas être compris sans tenir compte des excès autoritaristes du régime franquiste. La stratégie etarra de guerre révolutionnaire, notamment la spirale action/ répression, est ainsi directement liée à la violence du franquisme des années 1960. Ensuite, à partir des années 1970, l'ETA cessera progressivement d'être " une organisation politique disposant d'armes et de tout ce dont elle pouvait avoir besoin [...]" " pour devenir "une organisation militaire, et strictement militaire ${ }^{2} »$. Ignacio Domínguez, rédacteur en chef de l'agence Vasco Press, décrit l'épisode qui a duré de 1977 à 2002, illustrant les oscillations entre l'action armée et les solutions politiques de pacification. Sa contribution a la qualité de remettre en lien le rôle de l'ensemble des acteurs politiques, les partis nationalistes basques et nationaux (espagnols), avec leur volonté de trouver des résolutions au conflit. Vient ensuite une première conclusion de Patxo Unzueta, ancien dirigeant de l'ETA, qui revient sur l'omniprésence de l'organisation terroriste dans la détermination des orientations de la vie politique basque-espagnole, négligeant cependant le rôle des acteurs présents à Madrid. Antonio Elorza, dans sa conclusion, continue de fustiger le nationalisme basque, qu'il tient pour coupable de ne pas vouloir en finir avec l'ETA. Il conserve une vision parcellaire à la fois du mouvement des assemblées municipales basques, des manifestations de soutien auprès des victimes des attentats, et des événements de la vie politique basque en général. En un mot, ETA, une histoire, aurait dû s'intituler ETA, des histoires tant il est vrai que les contributions sont très inégales. Malheureusement, pour un lecteur non averti, le manque de rigueur qui apparaît parfois ne contribue pas toujours à présenter la "question basque " sous un angle incontestablement explicatif.

8 Le livre de Fernando Reinares, Patriotas de la muerte ("Patriotes de la mort »), nous offre une tout autre approche de l'ETA. D'ailleurs, il s'intéresse moins à l'ETA qu'à ceux qui ont milité dans l'ETA, comme le suggère le sous-titre de l'ouvrage. Son investigation cherche à comprendre les raisons qui ont amené essentiellement des jeunes garçons, d'une vingtaine d'années, pour la moitié d'entre eux originaires de la province du Guipúzcoa, à nourrir les rangs de l'organisation terroriste durant les années 1977 à 1997. Leurs motivations idéologiques mêlaient au départ nationalisme basque et préoccupations 
sociales marxisantes. Et, pour l'essentiel de ces etarras (membres de l'ETA), la violence politique apparaît surtout comme un moyen de parvenir à lutter contre un environnement politique qu'ils jugeaient alors intolérable, contre un système policier et répressif producteur de «haine ». Il s'agit d'un travail de fond considérable, effectué à partir des documents mis à la disposition de son enquête par l'Audiencia nacional (la plus haute instance juridique espagnole), poursuivi par une série d'entretiens auprès d'une soixantaine d'etarras (dont seulement quarante-sept d'entre eux seront retenus). Le travail est méthodique, rigoureux, il s'appuie sur les trajectoires de plus de six cents personnes répertoriées dans les dossiers d'instruction. Le travail est à la fois quantitatif et qualitatif. Tout au long des thématiques développées, Fernando Reinares prend le soin de relativiser les points de vue, argumentant les interprétations données à l'aide d'extraits d'entretiens équilibrés pour étayer son interprétation.

9 Face à un tel sujet, et l'ouvrage de Jean Chalvidant l'illustre bien, il est parfois difficile de trouver l'empathie nécessaire pour expliquer les choses. Fernando Reinares ne porte pas à proprement parler de jugement. Toutefois, comme il l'indique dans le prologue, par son statut tout à la fois de professeur d'université et de citoyen, il cherche à dénoncer le recours aux armes, en particulier quand celui-ci sert à défendre un nationalisme ethnique, « de passemontagnes et de txapela ("béret basque") », comme il aime à le répéter. En cela, c'est un ouvrage militant. Le titre de l'ouvrage, Patriotes de la mort, issu d'une annotation faite dans un dossier d'instruction par un procureur anonyme, tout comme l'expression «de passe-montagnes et de txapela» reviennent constamment pour qualifier les membres de l'ETA, devenant une rengaine exclusive et de fait, une maladresse de style. En dehors de ces lourdeurs de forme, les recoupements biographiques qu'il présente nous permettent néanmoins de caractériser ces individus qui ont intégré l'ETA. Ils offrent une grille d'analyse très intéressante sur les processus de socialisation politique (le sexe, l'age, l'origine géographique, les structures familiales, la formation idéologique, les contextes politiques et sociaux, les motivations individuelles, etc.). Indirectement, cet ouvrage démontre la complexité de l'ETA, qui a longtemps trouvé dans ses maillages sociaux le soutien populaire qui en explique la durée. De la même manière, la violence démesurée des forces de sécurité de l'Etat espagnol sous la dictature et leur manque de professionnalisme durant les premières années de la démocratie sont bien retranscrits à travers les témoignages. Tout au long de son ouvrage, F. Reinares décrit assez exhaustivement les raisons qui ont poussées les recrues de l'ETA à prendre les armes, et, ce faisant, il nous aide incontestablement à mieux la comprendre. 


\section{NOTES}

1.. Garmendia J. M., ETA, une histoire, op. cit., p. 257.

2.. Ibid.

3.. Reinares F., op. cit., pp. 16-17.

INDEX

Index géographique : Espagne

Mots-clés : guérillas et organisations clandestines, séparatismes/ autonomismes Thèmes : ETA

\section{AUTEUR}

JEAN-MARIE IZQUIERDO

Jean-Marie Izquierdo est chercheur associé au CERVL-Sciences Po Bordeaux. 